\title{
STRONGLY OMNIPRESENT OPERATORS: GENERAL CONDITIONS AND APPLICATIONS TO COMPOSITION OPERATORS
}

\author{
L. BERNAL-GONZÁLEZ, M. C. CALDERÓN-MORENO \\ and K.-G. GROSSE-ERDMANN
}

(Received 13 November 2000; revised 13 March 2001)

Communicated by P. C. Fenton

\begin{abstract}
This paper studies the concept of strongly omnipresent operators that was recently introduced by the first two authors. An operator $T$ on the space $H(G)$ of holomorphic functions on a complex domain $G$ is called strongly omnipresent whenever the set of $T$-monsters is residual in $H(G)$, and a $T$-monster is a function $f$ such that $T f$ exhibits an extremely 'wild' behaviour near the boundary. We obtain sufficient conditions under which an operator is strongly omnipresent, in particular, we show that every onto linear operator is strongly omnipresent. Using these criteria we completely characterize strongly omnipresent composition and multiplication operators.
\end{abstract}

2000 Mathematics subject classification: primary 30E10; secondary 30H05, 46E10, 47B38.

Keywords and phrases: holomorphic function, $T$-monster, residual set, strongly omnipresent operator, dense range, locally dense range, locally stable operator, composition operator, left-composition operator, multiplication operator.

\section{Introduction}

Inspired by the notion of holomorphic monsters as introduced and developed by Luh [8] and the third author [7, Kapitel 3] (see also [9, 10, 14]), the first two authors have recently introduced the concept of $T$-monsters [3], which is associated to a (not necessarily linear) operator $T$ on the space $H(G)$ of holomorphic functions on a domain $G$ in $\mathbb{C}$. Roughly speaking, a $T$-monster is a holomorphic function whose image under $T$ has an extremely 'wild' behaviour near the boundary.

The work of the first two authors has been partially supported by DGES grant PB96-1348 and the Junta de Andalucía.

(C) 2002 Australian Mathematical Society $1446-7887 / 2000 \$ A 2.00+0.00$ 
In [3] the concept was defined for $G \neq \mathbb{C}$ in order that the finite boundary be non-empty, but the interested reader can easily check, using chordal distances, that all proofs can be adapted to the case where the boundary point under consideration is the point of infinity. Consequently, we redefine the concept of $T$-monsters and its associated notion of strongly omnipresent operators [3, Section 2] (see also [2] for the weaker concept of omnipresent operators) in the following way.

Throughout this paper, $G$ will be a domain in $\mathbb{C}$ and $\partial G$ will denote its boundary taken in the extended complex plane $\mathbb{C}_{\infty}=\mathbb{C} \cup\{\infty\}$. By $\mathbb{D}$ we denote the open unit disk. An operator always refers to a continuous (not necessarily linear) mapping.

DEFINITION 1.1 ([3]). (a) A function $f \in H(G)$ is a holomorphic monster if it satisfies the following universality property:

$(U)$ for each $g \in H(\mathbb{D})$ and each $t \in \partial G$ there exists a sequence $\left(\tau_{n}\right)$ of affine linear transformations with $\tau_{n}(z) \rightarrow t(n \rightarrow \infty)$ uniformly on $\mathbb{D}$ and $\tau_{n}(\mathbb{D}) \subset G$ $\left(n \in \mathbb{N}\right.$ ) such that $f\left(\tau_{n}(z)\right) \rightarrow g(z)$ as $n \rightarrow \infty$ locally uniformly in $\mathbb{D}$.

(b) Let $T: H(G) \rightarrow H(G)$ be an operator. Then a function $f \in H(G)$ is a $T$ monster if $T f$ is a holomorphic monster. The set of $T$-monsters is denoted by $\mathscr{M}(T)$.

It is not difficult to see that in the case when the point of infinity is an isolated point in $\partial G$ this notion, in general, is strictly stronger than the one given in [3]; this is so, for example, if $T$ is the identity operator.

See below for a comparison with Luh's holomorphic monsters [8].

For the closely related notion of strongly omnipresent operators we need some more notation. We denote by $O(\partial G)=\left\{V \subset \mathbb{C}_{\infty}: V\right.$ is open and $\left.V \cap \partial G \neq \emptyset\right\}$ the set of all open subsets of $\mathbb{C}_{\infty}$ that meet the boundary of $G$. If $A \subset \mathbb{C}$ then $\bar{A}$ represents the closure of $A,\|f\|_{A}:=\sup _{z \in A}|f(z)|$, where $f$ is a complex function defined on $A$, and $L T(A)$ is the set of all affine linear transformations $\tau, \tau(z)=a z+b$, such that $\tau(\mathbb{D}) \subset A$.

DEFINITION 1.2 ([3]). An operator $T: H(G) \rightarrow H(G)$ is strongly omnipresent if for all $g \in H(\mathbb{D}), \varepsilon>0, r \in(0,1)$ and $V \in O(\partial G)$ the set $U(T, g, \varepsilon, r, V):=\{f \in$ $H(G)$ : there exists some $\tau \in L T(V \cap G)$ such that $\left.\|(T f) \circ \tau-g\|_{r \overline{\mathbf{D}}}<\varepsilon\right\}$ is dense in $H(G)$.

Again, in contrast to [3] we have here allowed the point of infinity as a boundary point. As in [3, Theorem 2.2] it is easy to prove that $T$ is strongly omnipresent if and only if the set $\mathscr{M}(T)$ of $T$-monsters is residual, that is, its complement in $H(G)$ is of first category.

As a consequence, every new strongly omnipresent operator $T$ yields a wealth of new universal functions: there is then a residual set of holomorphic functions $f$ so that 
each function $T f$ is a holomorphic monster, that is, each $T f$ satisfies the universality property $(U)$ stated in Definition 1.1.

Examples of strongly omnipresent operators are provided in [3, Sections 3-4]. Specifically, if $\Phi(z)=\sum_{j=0}^{\infty} a_{j} z^{j}$ is a non-zero entire function of subexponential type then the associated linear differential operator $\Phi(D)=\sum_{j=0}^{\infty} a_{j} D^{j}$ on $H(G)$ is strongly omnipresent. For $G=\mathbb{C}$ this result even holds for all entire functions $\Phi$ of exponential type. Here $D$ is the differentiation operator $D f=f^{\prime}, D^{0}=I$ is the identity operator and $D^{j+1}=D \circ D^{j}$. Furthermore, if $G$ is a simply connected domain, $a \in G, \lambda \in \mathbb{C}$ and $h$ is a non-zero entire function of exponential type then the integral operator $T$ on $H(G)$ defined by

$$
T f(z)=\lambda f(z)+\int_{a}^{z} h(z-t) f(t) d t \quad(z \in G)
$$

is strongly omnipresent. In particular, if $\Psi(z)=\sum_{j=0}^{\infty} a_{j} z^{j}$ is any non-zero function that is holomorphic at 0 then the corresponding linear antidifferential operator $\Psi\left(D_{a}^{-1}\right)=\sum_{j=0}^{\infty} a_{j} D_{a}^{-j}$ on $H(G)$ is strongly omnipresent. Here $D_{a}^{0}=I$ and, for each $j \in \mathbb{N}, D_{a}^{-j} f(f \in H(G))$ denotes the unique antiderivative $F$ of $f$ of order $j$ such that $F^{(k)}(a)=0(k \in\{0,1, \ldots, j-1\})$.

We note that the holomorphic monsters in the sense of Luh [8] are holomorphic functions that are simultaneously $D^{j}$ - and $D_{a}^{-j}$-monsters for all $j \in \mathbb{N}_{0}$. Since the intersection of countably many residual sets is again residual, the existence of Luhmonsters is thus a direct consequence of the strong omnipresence of each of the operators $D^{j}$ and $D_{a}^{-j}, j \in \mathbb{N}_{0}$.

Our aim in this paper is twofold. In Section 2 we derive conditions that guarantee that an operator is strongly omnipresent. This will be done in various ways. First we show how to construct new strongly omnipresent operators from known ones. As an application we will see that every onto linear operator is strongly omnipresent. Next we study the problem under which conditions the existence of a single $T$-monster suffices to make $T$ strongly omnipresent. Finally we derive some workable conditions under which a general operator is strongly omnipresent.

In Section 3 we apply these results to furnish new examples of strongly omnipresent operators that are substantially different from differential and antidifferential operators; specifically, we characterize the strongly omnipresent (left- and right-) composition operators and the strongly omnipresent multiplication operators.

\section{Looking for monsters}

We begin our investigation into the existence of monsters by constructing new strongly omnipresent operators from known ones. Before we do this we note the 
following two facts. Let $T$ be an operator on $H(G), \varepsilon>0, g \in H(\mathbb{D}), r \in(0,1)$ and $V \in O(\partial G)$. Then we have:

(1) $U(T, g, \varepsilon, r, V)=\{f \in H(G)$ : there exists some non-constant $\tau \in L T(V \cap G)$ such that $\left.\|(T f) \circ \tau-g\|_{r \overline{\mathrm{D}}}<\varepsilon\right\}=\{f \in H(G)$ : there exists some non-constant $\tau \in L T(V \cap G)$ such that $\left.\left\|T f-g \circ \tau^{-1}\right\|_{\tau(r \overline{\mathrm{D}})}<\varepsilon\right\}$. The first equality is accomplished by a simple continuity argument.

(2) $\mathscr{M}(T)=\bigcap_{g, \varepsilon, r, V} U(T, g, \varepsilon, r, V)$, where, in fact, it suffices to only consider countable dense subsets of $g, \varepsilon, r$ and a suitable collection of countably many sets $V$ that cover $\partial G$; in addition, each set $U(T, g, \varepsilon, r, V)$ is open. Hence $\mathscr{M}(T)$ is always a $G_{\delta}$-subset. Consequently it is residual if and only if it is dense, and if and only if $T$ is strongly omnipresent. For details see the proof of Theorem 2.2 in [3].

To start with, we state without proof the following result, which follows trivially from the definition of $T$-monster.

Proposition 2.1. Let $T, S: H(G) \rightarrow H(G)$ be operators. Then we have $S^{-1}(\mathscr{M}(T))=\mathscr{M}(T S)$. In particular,

(a) if $\mathscr{M}(T) \neq \emptyset$ and $S$ is onto then $\mathscr{M}(T S) \neq \emptyset$;

(b) if $\mathscr{M}(T S) \neq \emptyset$ then $\mathscr{M}(T) \neq \emptyset$.

THEOREM 2.2. Let $T, S: H(G) \rightarrow H(G)$ be operators such that $T$ is strongly omnipresent and $S$ is linear and onto. Then TS is strongly omnipresent.

PROOF. We have to prove that $\mathscr{M}(T S)$ is residual, hence, by the preceding proposition, that $S^{-1}(\mathscr{M}(T))$ is residual. But $\mathscr{M}(T)$ is always a $G_{\delta}$-subset, so $S^{-1}(\mathscr{M}(T))$ is also a $G_{\delta}$-subset because $S$ is continuous. It remains to see that $S^{-1}(\mathscr{M}(T))$ is dense. Since $\mathscr{M}(T)$ is dense, given a non-empty open subset $A$ in $H(G)$, we obtain that $\mathscr{M}(T) \cap S A \neq \emptyset$ because $S A$ is open due to the Open Mapping Theorem (recall that $S$ is linear and onto and that $H(G)$ is an F-space). Hence $S^{-1}(\mathscr{M}(T)) \cap A \neq \emptyset$. Thus, $S^{-1}(\mathscr{M}(T))$ is dense, as required.

COROLLARY 2.3. Every onto linear operator on $H(G)$ is strongly omnipresent.

PROOF. Apply Theorem 2.2 to $T=I$, the identity operator, which is strongly omnipresent: take $\Phi(z) \equiv z$ in the example given in the introduction.

For example, we know that for each $N \in \mathbb{N}$ the antidifferentiation operator $D_{a}^{-N}$ of order $N$ is strongly omnipresent on $H(G)$ (take $\Psi(z) \equiv z^{N}$ in the example given in the Introduction), where $G$ is a simply connected domain and $a$ is a fixed point in $G$. Since the differentiation operator $D$ (and so $D^{N}$ ) is onto on $H(G)$ due to simple 
connectedness we can conclude that the operator $R_{N, a}$ on $H(G)$ given by

$$
R_{N, a} f(z):=f(z)-\sum_{j=0}^{N-1} \frac{f^{(j)}(a)}{j !}(z-a)^{j},
$$

that is, the value at $z$ of Taylor's remainder of order $N$ of $f$ at $a$, is strongly omnipresent. Indeed, take $T=D_{a}^{-N}$ and $S=D^{N}$ in Theorem 2.2. Note that neither $D_{a}^{-N}$ nor $R_{N, a}$ is onto; they do not even have dense range.

In particular we obtain the following application in the case $G=\mathbb{C}$.

COROLLARY 2.4. There is an entire function $f(z)=\sum_{n=0}^{\infty} a_{j} z^{j}$ such that each of its Taylor series remainders $R_{N} f(z):=\sum_{j=N}^{\infty} a_{j} z^{j}\left(N \in \mathbb{N}_{0}\right)$ is a holomorphic monster.

It is evident that the sum $T+S$ of two strongly omnipresent operators need not be strongly omnipresent: take, for instance, $T=I, S=-I$. On the other hand, a non-zero multiple $\lambda T, \lambda \neq 0$, of a strongly omnipresent operator $T$ is trivially strongly omnipresent. The next result shows that if $T$ is strongly omnipresent and $S$ is an operator that behaves well near the boundary then their sum $T+S$ generates a strongly omnipresent operator, and a similar result is true for the product $T \cdot S$ given by $(T \cdot S) f=T f \cdot S f$.

THEOREM 2.5. Let T, $S$ be two operators on $H(G)$. Assume that there exists a dense subset $\Gamma$ in $\partial G$ such that, for every $t \in \Gamma$ and for every $f \in H(G)$, there exists $\lim _{z \rightarrow t}(S f)(z) \in \mathbb{C}$ (respectively $\left.\mathbb{C} \backslash\{0\}\right)$. Then $\mathscr{M}(T) \subset \mathscr{M}(T+S)$ (respectively $\mathscr{M}(T \cdot S)$ ). In particular, $T+S$ (respectively $T \cdot S$ ) is strongly omnipresent if $T$ is.

PROOF. Using [3, Lemma 2.1], the fact that $\mathscr{M}(T) \subset \mathscr{M}(T+S)$ (respectively $\mathscr{M}(T) \subset \mathscr{M}(T \cdot S))$ is straightforward from the definition of monster. The details are left to the reader.

As an example of an application of Theorem 2.5 we may consider again the Taylor remainder operator $R_{N, a}$ (given after Corollary 2.3) acting on $H(G)$, where $G$ is any domain such that the point of infinity is not an isolated point of $\partial G$. Indeed, we can write $R_{N, a}=T+S$ with $T=I$, the identity operator, and

$$
S f(z)=-\sum_{j=0}^{N-1} \frac{f^{(j)}(a)}{j !}(z-a)^{j} .
$$

It is evident that $S$ is 'well-behaved' on $\Gamma:=(\partial G) \backslash\{\infty\}$.

The next result provides us with a condition that guarantees the existence of a residual set of monsters by assuming the existence of at least one monster. For future reference, we isolate this condition and introduce the notion of (local) stability of an operator. 
DEFINITION 2.1. Let $T: H(G) \rightarrow H(G)$ be an operator.

(a) Let $K$ be a compact subset of $G$ and $B$ a closed ball contained in $G$. Then $T$ is $K$-stable in $B$ if for each $f \in H(G)$ and $\varepsilon>0$ there exist a closed ball $B^{\prime} \subset G \backslash K$ and $\delta>0$ such that for all $g \in H(G)$

$$
\|f-g\|_{B^{\prime}}<\delta \quad \text { implies that }\|T f-T g\|_{B}<\varepsilon \text {. }
$$

(b) We say that $T$ is locally stable near $\partial G$ if for each compact subset $K$ of $G$ there exists a compact subset $M$ of $G$ such that for each closed ball $B \subset G \backslash M, T$ is $K$-stable in $B$.

(c) We say that $T$ is somewhere locally stable near $\partial G$ if for each compact subset $K$ of $G$ and each $V \in O(\partial G)$ there exists a closed ball $B \subset V \cap G$ such that $T$ is $K$-stable in $B$.

For the sake of brevity we will usually take the qualification 'near $\partial G$ ' for granted. It is clear that every locally stable operator is somewhere locally stable. In fact, every locally stable operator $T$ has the property that for each compact subset $K$ of $G$ and each $t \in \partial G$ there is an open neighbourhood $V$ of $t$ such that for each closed ball $B \subset V \cap G, T$ is $K$-stable in $B$.

By using Cauchy's integral formula for derivatives, it is straightforward to verify that each differential operator $\Phi(D)$, where $\Phi$ is an entire function of subexponential type, is locally stable; in fact we can always take concentric closed balls $B, B^{\prime}$ with $\operatorname{radius}(B)<\operatorname{radius}\left(B^{\prime}\right)$. Another example of a locally stable operator is the rotation operator $R_{\alpha}(\alpha \in[0,2 \pi))$ on $H(\mathbb{D})$ defined by $R_{\alpha} f(z)=f\left(z e^{i \alpha}\right)$. Further examples will be given in Section 3.

THEOREM 2.6. Let $T$ be an operator on $H(G)$ that is locally stable near $\partial G$. If $\mathscr{M}(T) \neq \emptyset$ then $T$ is strongly omnipresent.

PROOF. Fix $g \in H(\mathbb{D}), \varepsilon>0, r \in(0,1)$ and $V \in O(\partial G)$. We are going to show that $U(T, g, \varepsilon, r, V)$ is dense in $H(G)$. To see this, fix a basic open subset

$$
D\left(h, K, \varepsilon_{1}\right)=\left\{f \in H(G):\|f-h\|_{K}<\varepsilon_{1}\right\}
$$

of $H(G)$, where $K$ is a compact subset of $G$ such that each connected component of $\mathbb{C}_{\infty} \backslash K$ contains at least one connected component of $\mathbb{C}_{\infty} \backslash G, h \in H(G)$ and $\varepsilon_{1}>0$. Now let $f$ be a fixed $T$-monster. Then there exists a non-constant affine linear transformation $\tau \in L T(G \cap V)$ such that

$$
\left\|T f-g \circ \tau^{-1}\right\|_{B}<\varepsilon / 2,
$$


where $B:=\tau(r \overline{\mathbb{D}})$. We can assume that $B \subset G \backslash M$, where $M$ is the compact subset of $G$ given by the definition of local stability. Hence there exist a closed ball $B^{\prime} \subset G \backslash K$ and $\delta>0$ such that, for all $\varphi \in H(G)$,

$$
\|\varphi-f\|_{B^{\prime}}<\delta \text { implies that }\|T \varphi-T f\|_{B}<\varepsilon / 2 .
$$

Consider the compact set $L:=K \cup B^{\prime}$. Then each connected component of the complement of $L$ contains at least one component of the complement of $G$ because $K$ has this property and $K \cap B^{\prime}=\emptyset$. Pick open subsets $G_{1}, G_{2} \subset G$ with $G_{1} \cap G_{2}=\emptyset$ and $K \subset G_{1}, B^{\prime} \subset G_{2}$. Denote $G_{0}=G_{1} \cup G_{2}$. Hence $G_{0}$ is open and $L \subset G_{0} \subset G$. Define the function $F: G_{0} \rightarrow \mathbb{C}$ by

$$
F(z)= \begin{cases}h(z), & \text { if } z \in G_{1} \\ f(z), & \text { if } z \in G_{2} .\end{cases}
$$

Then $F \in H\left(G_{0}\right)$ and an application of Runge's theorem [13, Chapter 13] yields the existence of a rational function $f_{1}$ with poles outside $G$ such that

$$
\left\|f_{1}-F\right\|_{L}<\min \left\{\delta, \varepsilon_{1}\right\}
$$

Thus, $f_{1} \in H(G)$ with

$$
\left\|f_{1}-h\right\|_{K}<\varepsilon_{1}
$$

and $\left\|f_{1}-f\right\|_{B^{\prime}}<\delta$. From (2) we obtain

$$
\left\|T f_{1}-T f\right\|_{B}<\varepsilon / 2 .
$$

Then (1) and (4) lead us to

$$
\left\|T f_{1}-g \circ \tau^{-1}\right\|_{B}<\varepsilon .
$$

Summarizing, (3) and (5) tell us that $f_{1} \in U(T, g, \varepsilon, r, V) \cap D\left(h, K, \varepsilon_{1}\right)$. Consequently, $U(T, g, \varepsilon, r, V)$ is dense, as required.

We state another condition under which the existence of a single monster guarantees the existence of a large supply of monsters: We assume that, on a dense set of functions, $T$ is well-behaved near the boundary. This time linearity of $T$ is needed.

THEOREM 2.7. Let $T$ be a linear operator on $H(G)$ with $\mathscr{M}(T) \neq \emptyset$. If there exists a dense subset $\mathscr{D}$ in $H(G)$ with the property that for each $h \in \mathscr{D}$ there is a dense subset $\Gamma_{h}$ in $\partial G$ such that, for all $t \in \Gamma_{h}$, there exists $\lim _{z \rightarrow t}(T h)(z) \in \mathbb{C}$, then $T$ is strongly omnipresent.

ProOF. Pick a $T$-monster $f$. Then $f+\mathscr{D}$ is dense in $H(G)$. Fix $h \in \mathscr{D}$ and consider its corresponding set $\Gamma_{h} \subset \partial G$. Given $t \in \Gamma_{h}$ and $g \in H(\mathbb{D})$, define the 
function $g_{1}(z):=g(z)-L(t)$, where $L(t):=\lim _{z \rightarrow t}(T h)(z) \in \mathbb{C}$. Then $g_{1} \in H(\mathbb{D})$ and there exist two sequences of complex numbers $\left(a_{n}\right),\left(b_{n}\right)$ with $a_{n} z+b_{n} \rightarrow t$ $(n \rightarrow \infty)$ uniformly on $\mathbb{D}$ and $a_{n} z+b_{n} \in G$ for all $n \in \mathbb{N}$ and all $z \in \mathbb{D}$ such that

$$
(T f)\left(a_{n} z+b_{n}\right) \rightarrow g_{1}(z) \quad(n \rightarrow \infty)
$$

locally uniformly in $\mathbb{D}$. But we also have that $(T h)\left(a_{n} z+b_{n}\right) \rightarrow L(t)(n \rightarrow \infty)$ locally uniformly in $\mathbb{D}$ (in fact, uniformly on the whole $\mathbb{D}$ ). Therefore, by linearity, $(T(f+h))\left(a_{n} z+b_{n}\right) \rightarrow g(z)(n \rightarrow \infty)$ in the same manner. In view of [3, Lemma 2.1] this implies that $f+\mathscr{D} \subset \mathscr{M}(T)$, so that $\mathscr{M}(T)$ is dense.

For instance, the condition in the above theorem is satisfied by a differential operator $\Phi(D)$ and by a finite order antidifferential operator $\Psi\left(D^{-1}\right)$ whenever $G$ is a simply connected domain with $G \neq \mathbb{C}$ : just let $\mathscr{D}$ be the set of all polynomials and let $\Gamma_{h}$ be the finite boundary of $G$ for all $h \in \mathscr{D}$.

Although the last two theorems give mild conditions under which $\mathscr{M}(T) \neq \emptyset$ implies that $T$ is strongly omnipresent the following example shows that this implication does not hold in general. Recall that $I$ denotes the identity operator.

EXAMPLE 2.8. Consider the operator $T: H(\mathbb{D}) \rightarrow H(\mathbb{D})$ given by

$$
T f(z)=\psi\left(\frac{z}{1+|f(0)|}\right) \quad(z \in \mathbb{D}),
$$

where $\psi$ is a fixed holomorphic monster in $\mathbb{D}$. Then $f$ is a $T$-monster if and only if $f(0)=0$. Hence $T$-monsters exist, but they only form a set of first category.

This example gives a partial solution to a problem posed in [3]. Unfortunately the problem remains open for linear operators:

If $\mathscr{M}(T) \neq \emptyset$, is $T$ always strongly omnipresent?

Next, we want to derive practicable conditions on an operator that guarantee its strong omnipresence. Here the range, or rather the local ranges, of $T$ will play an important role. This leads us to the following definition.

DEFINITION 2.2. Let $T: H(G) \rightarrow H(G)$ be an operator.

(a) Let $U \subset G$ be an open ball. Then $T$ has dense range in $U$ if the operator

$$
T_{U}: H(G) \rightarrow H(U), \quad f \mapsto T_{U} f=(T f)_{\left.\right|_{v}}
$$

has dense range.

(b) We say that $T$ has locally dense range near $\partial G$ if there exists a compact subset $M$ of $G$ such that for each open ball $U \subset G \backslash M, T$ has dense range in $U$. 
(c) We say that $T$ has somewhere locally dense range near $\partial G$ if for each $V \in$ $O(\partial G)$ there exists an open ball $U \subset V \cap G$ such that $T$ has dense range in $U$.

As before, we will usually take the qualification 'near $\partial G$ ' for granted.

It is clear that every operator with dense range has locally dense range and that every operator with locally dense range has somewhere locally dense range.

Up to now, all the known examples of strongly omnipresent operators satisfy one of the previous properties. For instance, recall that every non-zero differential operator $\Phi(D)$ has dense range whenever $G$ is simply connected: indeed, $\Phi(D)$ is onto on the space of entire functions $H(\mathbb{C})$ (see $[6,11])$ and $H(\mathbb{C})$ is dense in $H(G)$. In fact, in any domain $G, \Phi(D)$ always has locally dense range. Also the antidifferential operator $D_{a}^{-N}$ has locally dense range near the boundary.

One could believe that there exists some characterization of strongly omnipresent operators in terms of the size of the range. That is not true, however, as we are going to see in the next example. We can construct strongly omnipresent operators with 'very small' range.

EXAMPLE 2.9. Let $G \subset \mathbb{C}$ be a domain with $0 \in G$ and consider the operator $T: H(G) \rightarrow H(G)$ defined by $T f=f(0) \psi$, where $\psi$ is a fixed holomorphic monster in $G$. Then $f$ is a $T$-monster if and only if $f(0) \neq 0$. Hence $T$ is strongly omnipresent, but $T$ has 1-dimensional range.

On the other hand, having 'large' range need not imply strong omnipresence. As the following example shows, there exist operators with dense range but without any monster.

EXAMPLE 2.10. Let $G=\mathbb{D}, r \in(0,1)$ fixed and $T: H(\mathbb{D}) \rightarrow H(\mathbb{D})$ the operator given by $T f(z)=f(r z)(z \in \mathbb{D})$. It is obvious that all the polynomials lie in the range of $T$, so $T$ has dense range. But $\mathscr{M}(T)=\emptyset$ because $T f$ is continuous up to the boundary for all $f \in H(\mathbb{D})$.

In spite of the fact that these examples preclude any direct relationship between the range of an operator and its behaviour near the boundary our next result furnishes a sufficient condition for an operator with locally dense range to have many monsters: we will meet the notion of local stability again.

In the following, let $A^{0}$ denote the interior of the set $A$.

THEOREM 2.11. Let $T: H(G) \rightarrow H(G)$ be an operator such that for each compact subset $K \subset G$ and each set $V \in O(\partial G)$ there is some closed ball $B \subset V \cap G$ with

(a) $T$ has dense range in $B^{0}$,

(b) $T$ is $K$-stable in $B$.

Then $T$ is strongly omnipresent. 
ProOF. As in the beginning of the proof of Theorem 2.6 and with the same meaning, fix $g, \varepsilon, r, V, h, K$ and $\varepsilon_{1}$. Let $B \subset V \cap G$ be the closed ball given by the hypothesis and consider an affine linear transformation $\tau$ such that $\tau(\mathbb{D})=B^{0}$. Then $\tau \in L T(V \cap G)$ and $g \circ \tau^{-1} \in H\left(B^{0}\right)$. Therefore, by (a), there exists a function $f \in H(G)$ such that

$$
\left\|T f-g \circ \tau^{-1}\right\|_{\tau(r \overline{\mathbf{D}})}<\varepsilon / 2 .
$$

By (b), there exist a closed ball $B^{\prime} \subset G \backslash K$ and $\delta>0$ such that for all $\varphi \in H(G)$

$$
\|\varphi-f\|_{B^{\prime}}<\delta \quad \text { implies that }\|T \varphi-T f\|_{B}<\varepsilon / 2 .
$$

Now, an application of Runge's theorem (as in the proof of Theorem 2.6) leads us to the existence of a function $f_{1} \in H(G)$ with

$$
\left\|f_{1}-h\right\|_{K}<\varepsilon_{1}
$$

and $\left\|f_{1}-f\right\|_{B^{\prime}}<\delta$. So, by (7),

$$
\left\|T f_{1}-T f\right\|_{B}<\varepsilon / 2 .
$$

Now, (6), (9) and the fact that $\tau(r \overline{\mathbb{D}}) \subset B$ give us

$$
\left\|T f_{1}-g \circ \tau^{-1}\right\|_{\tau(r \overline{\mathbf{D}})} \leq\left\|T f_{1}-T f\right\|_{B}+\left\|T f-g \circ \tau^{-1}\right\|_{\tau(r(\overline{\mathbf{D}})}<\varepsilon .
$$

Hence, by (8) and (10) we have $f_{1} \in U(T, g, \varepsilon, r, V) \cap D\left(h, K, \varepsilon_{1}\right)$, and the proof is finished.

From the above theorem we obtain immediately the following.

COROLlARY 2.12. Let $T$ be an operator on $H(G)$ that has, near $\partial G$, either

- somewhere locally dense range and local stability; or

- locally dense range and somewhere local stability.

Then $T$ is strongly omnipresent.

Note that, in particular, if $T$ is an onto somewhere locally stable operator (not necessarily linear) then it is strongly omnipresent, compare with Corollary 2.3 .

In turn, we obtain again, independently, that the identity operator is strongly omnipresent.

\section{Composition and multiplication operators}

So far, the only concrete examples of strongly omnipresent operators are differential, antidifferential and integral operators, see [3]. In this section we will apply 
the results of the previous section to enlarge this supply by characterizing completely when a left- or right-composition operator or a multiplication operator is strongly omnipresent.

Let $H(G, G)=\{\varphi \in H(G): \varphi(G) \subset G\}$. Recall that for $\varphi \in H(G, G)$ the associated (right-)composition operator is the mapping $C_{\varphi}: H(G) \rightarrow H(G)$ defined by $C_{\varphi}(f)=f \circ \varphi$, which is in fact a linear operator on $H(G)$. Assume first that $\varphi \in \operatorname{Aut}(G)$ is an automorphism on $G$, that is, a one-to-one and onto function in $H(G, G)$. Then it is evident that $C_{\varphi}$ is an isomorphism from $H(G)$ onto itself and therefore strongly omnipresent by Corollary 2.3. But there are also plenty of selfmappings $\varphi \notin \operatorname{Aut}(G)$ that generate strongly omnipresent composition operators. It turns out that the corresponding characterization is purely topological. The key is that $\varphi$ must not 'forget' the boundary of $G$. Before establishing the result we isolate the appropriate topological condition.

(C) For every $V \in O(\partial G)$ the set $\varphi(V \cap G)$ is not relatively compact in $G$.

We state a number of equivalent versions of $(C)$.

$\left(\mathrm{C}_{1}\right)$ For every $V \in O(\partial G)$ the set $\partial G \cap \partial \varphi(V \cap G)$ is non-empty.

$\left(\mathrm{C}_{2}\right)$ For every $V \in O(\partial G)$ and every compact set $K \subset G$ there is an open ball $U \subset V \cap G$ with $\varphi(U) \cap K=\emptyset$.

$\left(C_{3}\right)$ For every $t \in \partial G$ we have that $S(\varphi, t) \cap \partial G \neq \emptyset$, where $S(\varphi, t)$ is the cluster set of $\varphi$ at the boundary point $t$ (for definition and properties of cluster sets, see for instance [5] and [12]).

THEOREM 3.1. Let $C_{\varphi}$ be the composition operator on $H(G)$ defined by $\varphi \in$ $H(G, G)$. Then the following assertions are equivalent:

(a) $C_{\varphi}$ is strongly omnipresent;

(b) $\mathscr{M}\left(C_{\varphi}\right)$ is non-empty;

(c) $\varphi$ satisfies $(\mathrm{C})$.

PROOF. It is trivial that (a) implies (b). Now assume that $\varphi$ does not satisfy (C). Then there is a compact set $K \subset G$ and an open set $V \in O(\partial G)$ with $\varphi(V \cap G) \subset K$. Hence, for every function $f \in H(G)$ we have that $f \notin U\left(C_{\varphi}, g, \varepsilon, r, V\right)$ if we choose $r \in(0,1)$ arbitrary, $\varepsilon=1$ and $g(z):=1+\|f\|_{K}(z \in \mathbb{D})$. It follows from Note 2 before Proposition 2.1 that $f$ cannot be a $C_{\varphi}$-monster. This shows that $\mathscr{M}\left(C_{\varphi}\right)=\emptyset$ and hence that (b) implies (c).

For the proof that (c) implies (a) we apply Theorem 2.11 . Let $K \subset G$ be a compact subset and $V \in O(\partial G)$. Then by property $\left(\mathrm{C}_{2}\right)$ there is an open ball $U \subset V \cap G$ with $\varphi(U) \cap K=\emptyset$. Since $\varphi$ is clearly non-constant we see that $\varphi^{\prime}$ is not identically zero on $U$ so that we can assume that $\varphi$ is one-to-one on $U$. We now choose closed balls $B^{\prime} \subset \varphi(U)$ and $B \subset U$ with $\varphi(B) \subset B^{\prime}$. Since $C_{\varphi} f=f \circ \varphi$ we see that 
$C_{\varphi}$ is $K$-stable in $B$. Also, $C_{\varphi}$ has dense range in $B^{0}$ because $\varphi: B^{0} \rightarrow \varphi\left(B^{0}\right)$ is a holomorphic bijection and $H(G)$ is dense in $H\left(\varphi\left(B^{0}\right)\right)$ by Runge's theorem; note that $\varphi\left(B^{0}\right)$ is a simply connected domain contained in $G$. Theorem 2.11 now implies the result.

COROLLARY 3.2. Let $\varphi$ be an entire function and $C_{\varphi}$ the associated composition operator on $H(\mathbb{C})$. Then the following assertions are equivalent:

(a) $C_{\varphi}$ is strongly omnipresent;

(b) $\mathscr{M}\left(C_{\varphi}\right)$ is non-empty;

(c) $\varphi$ is non-constant.

Proof. Here our domain is $G=\mathbb{C}$. Property (C) reads in this case as follows: For every $R>0$ the set $\varphi(|z|>R)$ is not bounded. This holds if and only if $\varphi$ is not bounded, hence, by Liouville's theorem, if and only if $\varphi$ is non-constant. It remains to apply Theorem 3.1.

EXAMPLE 3.3. By Corollary 3.2 there is an entire function $f$ so that the entire function $f \circ \exp$ is a holomorphic monster. More generally, given any sequence $\left(\varphi_{n}\right)$ of non-constant entire functions there is an entire function $f$ such that each of the functions $f \circ \varphi_{n}(n \in \mathbb{N})$ is a holomorphic monster.

We now consider left-composition operators. For this we need to assume that $\varphi$ is an entire function. Then the left-composition operator $L_{\varphi}: H(G) \rightarrow H(G)$ is defined by $L_{\varphi}(f)=\varphi \circ f$, which is only a linear operator if $\varphi$ is linear.

In this case the characterizing condition for strong omnipresence turns out to be in terms of the size of the range of $L_{\varphi}$.

(L) The operator $L_{\varphi}: H(\mathbb{D}) \rightarrow H(\mathbb{D})$ has dense range.

The following are useful equivalent variants of $(\mathrm{L})$. We leave the proof of the equivalence to the reader; suffice it to say that Runge's theorem is crucial, by which $H(\mathbb{C})$ is dense in $H(O)$ for every simply connected domain $O$.

$\left(\mathrm{L}_{1}\right)$ There exists a simply connected domain $O$ in $\mathbb{C}$ such that $L_{\varphi}: H(O) \rightarrow H(O)$ has dense range.

$\left(\mathrm{L}_{2}\right)$ For any domain $G$ and any simply connected domain $O \subset G, L_{\varphi}: H(G) \rightarrow$ $H(O)$ has dense range.

$\left(\mathrm{L}_{3}\right)$ The function $\varphi$ has an approximate right inverse in $H(\mathbb{C})$, that is, there is a sequence $\left(f_{n}\right)$ of entire functions such that $\varphi\left(f_{n}(z)\right) \rightarrow z$ locally uniformly in $\mathbb{C}$.

THEOREM 3.4. Let $L_{\varphi}$ be the left-composition operator on $H(G)$ defined by $\varphi \in$ $H(\mathbb{C})$. Then the following assertions are equivalent:

(a) $L_{\varphi}$ is strongly omnipresent; 
(b) $\mathscr{M}\left(L_{\varphi}\right)$ is non-empty;

(c) $\varphi$ satisfies $(\mathrm{L})$.

PROOF. It is trivial that (a) implies (b). If $f$ is an $L_{\varphi}$-monster then for any $g \in H(\mathbb{D})$ there is a sequence $\left(\tau_{n}\right)$ in $L T(G)$ such that $\varphi \circ\left(f \circ \tau_{n}\right)(z)=\left(L_{\varphi} f\right) \circ \tau_{n}(z) \rightarrow g(z)$ locally uniformly in $\mathbb{D}$. This shows that (L) holds, hence that (b) implies (c).

Now assume that (c) holds. Then $L_{\varphi}$ has locally dense range because, by $\left(L_{2}\right)$, for any open ball $U$ in $G$ the operator $L_{\varphi}: H(G) \rightarrow H(U)$ has dense range. Moreover, $L_{\varphi}$ is locally stable since the operator $L_{\varphi}: H(U) \rightarrow H(U)$ is always continuous. By Corollary $2.12, L_{\varphi}$ is strongly omnipresent, so that (a) holds.

It follows from this theorem that many left-composition operators $L_{\varphi}$ are strongly omnipresent. For example, whenever $\varphi$ is universal in the sense of Birkhoff [4], that is, if the set $\{\varphi(\cdot+a): a \in \mathbb{C}\}$ is dense in $H(\mathbb{C})$, then $\varphi$ clearly satisfies $\left(\mathrm{L}_{3}\right)$, and the set of these functions is known to be residual in $H(\mathbb{C})$. The same is true, more generally, for any holomorphic monster in $\mathbb{C}$. As a consequence of this and Corollary 3.2 we have the following.

EXAMPLE 3.5. Let $\varphi \in H(\mathbb{C})$ be a holomorphic monster. Then there is a residual set of entire functions $f$ such that both $f \circ \varphi$ and $\varphi \circ f$ are holomorphic monsters. Clearly, $f(z)=z$ is one such function.

On the other hand, it follows from Hurwitz's theorem [1, page 178] that only surjective functions $\varphi$ can satisfy $\left(\mathrm{L}_{3}\right)$, hence $(\mathrm{L})$. Moreover, surjectivity alone is not sufficient; for instance, the function $\varphi(z):=z^{2}$ does not satisfy $\left(\mathrm{L}_{3}\right)$. This follows from Rouché's theorem [1, page 153], because if $f_{n}^{2}(z) \rightarrow z$ locally uniformly in C then, for large $n, f_{n}^{2}$ would have exactly one zero in the unit disk, counting multiplicity, which is clearly absurd.

In contrast to these observations it should be noted that the operator $L_{\varphi}$ is always omnipresent when $\varphi$ is a non-constant entire function, see Theorem 1(c) of [2]. As a consequence there are omnipresent operators that are not strongly omnipresent. We show by an example that there are even linear operators with this property, thus answering a question posed in [3].

EXAMPLE 3.6. Let $G$ be a domain containing the origin and let $T: H(G) \rightarrow H(G)$ be the linear operator defined by $T f=f(0) e^{\psi}$, where $\psi$ is a fixed holomorphic monster. Then $T$ is omnipresent but not strongly omnipresent. For if $f(0) \neq 0$ then $T f$ has maximal cluster sets at every boundary point of $G$ because $\psi$ has this property and because the exponential function has dense range. Thus $T$ is omnipresent by [2, Theorem 1 (a)]. On the other hand, no function $f$ can be a $T$-monster. This follows from Hurwitz's theorem because $e^{\psi}$ omits the value 0 and hence cannot be used to approximate functions that have a zero but are not identically zero. 
We finally study multiplication operators. For $\psi \in H(G)$ the operator $M_{\psi}$ : $H(G) \rightarrow H(G)$ is defined by $M_{\psi} f(z)=\psi(z) f(z) \quad(z \in G)$. By Theorem 2.5, taking $T=I$, the identity operator, and $S$ the constant operator with value $\psi$, we know that $M_{\psi}$ is strongly omnipresent if $\psi$ extends to a continuous function on the boundary without zeros there. In fact, we can drop this extra condition.

THEOREM 3.7. If $\psi$ is non-zero then the multiplication operator $M_{\psi}$ is strongly omnipresent.

Proof. For any $\psi, M_{\psi}$ is obviously locally stable. Now let $V \in O(\partial G)$. If $\psi \neq 0$ there is an open ball $U \subset V \cap G$ such that $\psi$ has no zeros in $U$. Then it is clear that $M_{\psi}$ has dense range in $U$ by Runge's theorem. Hence $M_{\psi}$ also has somewhere locally dense range, which implies the result by Corollary 2.12 .

\section{References}

[1] L. V. Ahlfors, Complex analysis, 3rd edition (McGraw-Hill, London, 1979).

[2] L. Bernal-González, 'Omnipresent holomorphic operators and maximal cluster sets', Colloq. Math. 63 (1992), 315-322.

[3] L. Bernal-González and M. C. Calderón-Moreno, 'Holomorphic $T$-monsters and strongly omnipresent operators', J. Approx. Theory 104 (2000), 204-219.

[4] G. D. Birkhoff, 'Démonstration d'un théorème élémentaire sur les fonctions entières', C. R. Acad. Sci. Paris 189 (1929), 473-475.

[5] E. F. Collingwood and A. J. Lohwater, The theory of cluster sets (Cambridge University Press, Cambridge, 1966).

[6] L. Ehrenpreis, 'Mean periodic functions I', Amer. J. Math. 77 (1955), 293-328.

[7] K.-G. Grosse-Erdmann, 'Holomorphe Monster und universelle Funktionen', Mitt. Math. Sem. Giessen 176 (1987).

[8] W. Luh, 'Holomorphic monsters', J. Approx. Theory 53 (1988), 128-144.

[9] —_, 'Multiply universal holomorphic functions', J. Approx. Theory 89 (1997), 135-155.

[10] W. Luh, V. A. Martirosian and J. Müller, ' $T$-universal functions with lacunary power series', Acta Sci. Math. (Szeged) 64 (1998), 67-79.

[11] B. Malgrange, 'Existence et approximation des solutions des équations aux dérivées partielles et des équations de convolution', Ann. Inst. Fourier (Grenoble) 6 (1955/1956), 271-355.

[12] K. Noshiro, Cluster sets (Springer, Berlin, 1960).

[13] W. Rudin, Real and Complex Analysis, 2nd edition (Tata McGraw-Hill, Faridabad, 1974).

[14] I. Schneider, 'Schlichte Funktionen mit universellen Approximationseigenschaften', Mitt. Math. Sem. Giessen 230 (1997).

Departamento de Análisis Matemático

Facultad de Matemáticas, Apdo. 1160

Avenida Reina Mercedes

41080 Sevilla

Spain

e-mail: lbernal@us.es,mccm@us.es
Fachbereich Mathematik

Fernuniversität Hagen 58084 Hagen

Germany e-mail: kg.grosse-erdmann@ fernuni-hagen.de 